\title{
ORIGINAL RESEARCH \\ Local Anesthesia with Bupivacaine and Lidocaine for Vertebral Fracture Trial (LABEL): A Report of Outcomes and Comparison with the Investigational Vertebroplasty Efficacy and Safety Trial (INVEST)
}

W. Brinjikji

B.A. Comstock

L. Gray

D.F. Kallmes

\begin{abstract}
BACKGROUND AND PURPOSE: INVEST is a recently published double-blind placebo controlled randomized trial that demonstrated similar improvements in pain between blinded vertebroplasty and shamvertebroplasty groups. LABEL is a trial determining the efficacy of pain relief of an injection of lidocaine and bupivacaine at the site of painful osteoporotic vertebral compression fractures in unblinded patients. We compared outcomes from the unblinded LABEL trial with those of blinded control patients from the lead site of the INVEST, exploring the role of blinding on the benefit of local anesthesia infusion for painful vertebral compression fractures.
\end{abstract}

\begin{abstract}
MATERIALS AND METHODS: Nineteen patients with painful osteoporotic vertebral compression fractures underwent unblinded injection of lidocaine and bupivacaine at the site of painful osteoporotic vertebral compression fractures. Patients were given the option of undergoing vertebroplasty at any time following the procedure. Primary outcome measures were change in the RDQ and pain (at rest, with activity, and average 24-hour pain) at days 1 and 3 following the injection. Day 3 change in RDO scores and change in average 24-hour pain were compared for LABEL and INVEST control patients from the lead site $(n=16)$.
\end{abstract}

RESULTS: Among patients in the LABEL trial, we detected no significant improvement in RDQ scores, pain at rest, and average 24-hour pain at days 1 and 3, whereas pain with activity improved significantly at both time points. INVEST control patients from the lead site experienced significantly greater improvement in average pain during 24 hours at days $1(P=.03)$ and $3(P=.04)$ and significantly greater improvements in RDQ scores at day $3(P=.006)$ than patients from LABEL.

CoNCLUSIONS: An unblinded injection of local anesthesia is ineffective in treating pain from osteoporotic compression fractures. This suggests that factors other than local anesthesia were responsible for the observed improvement in the control group in INVEST.

ABBREVIATIONS: INVEST = Investigational Vertebroplasty Efficacy and Safety Trial; LABEL = Local Anesthesia with Bupivacaine and Lidocaine for Vertebral Fracture; RCT = randomized controlled trial; RDG = Roland-Morris Disability Questionnaire
$\mathbf{T}$ wo independent placebo-controlled RCTs recently demonstrated that patients allocated to either a full vertebroplasty or a "control intervention" in which the vertebroplasty procedure was simulated but no cement was infused achieved equivalent reductions in pain and improvements in back pain-related disability. ${ }^{1,2}$ In 1 of these 2 RCTs, INVEST, the simulated vertebroplasty included the infusion of subcutaneous lidocaine and periosteal bupivacaine, as is typically done in the vertebroplasty procedure. ${ }^{2}$

The outcomes from these RCTs suggest that the observed efficacy of the vertebroplasty procedure, instead of representing cement-mediated reduction in pain, may relate to other factors, including patients' expectations of benefit (the pla-

Received March 1, 2010; accepted after revision March 21

From the Mayo Medical School (W.B.) and Department of Radiology (L.G., D.F.K.), Mayo Clinic, Rochester, Minnesota; and Department of Biostatistics (B.A.C.), University of Washington, Seattle, Washington.

Paper previously presented at: American Society of Neuroradiology Annual Meeting and NER Foundation Symposium, May 17-20, 2010; Boston, Massachusetts.

Please address correspondence to David F. Kallmes, MD, Mayo Clinic, OL 1-115, 200 SW First St, Rochester, MN 55905; e-mail: kallmes.david@mayo.edu

DOI 10.3174/ajnr.A2145 cebo effect) or to the local administration of lidocaine and bupivacaine, or both. To further explore the relative impact of these potential factors, we carried out a prospective trial in which patients in an unblinded fashion underwent the local deposition of lidocaine and bupivacaine for treatment of painful osteoporotic compression fractures and we compared outcomes in this unblinded cohort with the blinded cohort allocated to the simulated vertebroplasty at the lead site in the INVEST.

\section{Materials and Methods}

\section{Study Subjects}

Consecutive patients presenting to our clinic for consideration of vertebroplasty for painful vertebral compression fractures were screened for enrollment between April 2009 and January 2010. The trial was called "LABEL." Institutional review board approval was obtained before the start of this Health Insurance Portability and Accountability Act-complaint study, and informed consent was obtained from all study participants. Inclusion criteria were the following: 1) age $>40$ years, 2 ) $\leq 4$ osteoporotic fractures of $<12$ months' 
duration, 3) pain score of at least 3 of ten, 4) English-speaking participants who had access to a telephone, 5) ability to give informed consent, and 6) a normalized International Normalized Ratio. Patients were excluded if they had local infection, tumor involvement of the vertebra to be treated, diagnosis of multiple myeloma, or were women of child-bearing potential.

\section{Patient Evaluation}

LABEL was an unblinded observational cohort study of patients presenting for consideration of vertebroplasty at our Neuroradiology Clinic. Patients were evaluated for appropriateness of vertebroplasty and were counseled that the true efficacy of the cement injection has not been proven. Patients were counseled that recent data suggest that a substantial portion of patients may benefit from local anesthesia without cement injection. At the time of consent, they were told that they could ask for and obtain a vertebroplasty at any time during follow-up.

\section{Collection of Baseline Variables}

Baseline data collection included age and sex. Outcome measures collected at baseline included the modified 23-question $\mathrm{RDQ}^{3-5}$ and a $0-10$ rating of average pain during 24 hours, pain with activity, and pain at rest.

\section{Procedural Characteristics}

After screening and consent signature, patients were escorted to a fluoroscopy room. They were instructed regarding routine postprocedural concerns, including delayed allergic reaction and bleeding. All procedures were performed under strict aseptic conditions. Patients were placed prone on a fluoroscopy table. Standard fluoroscopy was used for localization of the vertebral body/bodies to be treated (levels T4-L5). A 1-inch 25-gauge needle was used to raise a wheal of $0.25 \%$ lidocaine subcutaneously. A 10 -cm 25 -gauge spinal needle was placed into the skin at the site of the wheal and advanced under fluoroscopic guidance to the periosteum over the pedicle. This periosteum was then infiltrated with $6-7 \mathrm{~mL}$ of $0.25 \%$ bupivacaine. Injections were made at each fracture level. As is typical for our vertebroplasty practice, unipediculate injections were performed at each level.

\section{Follow-Up}

Follow-up outcome measures included RDQ score, average pain during 24 hours, pain with activity, pain at rest, and patient decision to undergo vertebroplasty. Patients enrolled in LABEL were followed at prescheduled intervals similar to the INVEST: days 1, 3, and 7, two weeks, 1 month, and 3 months. Follow-up in this trial continued until 3 months or the patient underwent vertebroplasty, whichever came first. Due to early crossover to vertebroplasty, we only reported on outcome measures observed through day 3 . If desired by the patient, he or she was prescheduled for vertebroplasty at the time of enrollment into the LABEL trial. These vertebroplasty procedures were typically prescheduled for 1-7 days following the initial evaluation.

\section{INVEST Control Group Data}

Only data from patients from the lead site of INVEST were included in this analysis. The study methods have been described previously. ${ }^{2,6}$ The inclusion criteria for these patients were similar to those of LABEL and were the following: 1) The patient must be 50 years of age or older; 2) He or she should have a diagnosis of 1-4 painful osteoporotic vertebral compression fractures between vertebral levels T4 and L5; 3) inadequate pain relief with standard medical therapy; 4) a rating for pain intensity of at least 3 on a $0-10$ scale; and 5) the fracture needed to be $<1$ year old. Exclusion criteria were evidence or suspicion of a neoplasm in the target vertebral body, retropulsion of bony fragments, concomitant hip fracture, active infection, and uncorrectable bleeding diatheses.

The INVEST control group underwent a blinded sham vertebroplasty with injection of lidocaine and bupivacaine. With the exception of the sham vertebroplasty procedures, the process of injection of local bupivacaine and lidocaine was identical to that of the LABEL study. The methods of blinding and injection of local anesthesia are described elsewhere. ${ }^{2,6}$

\section{Statistical Analysis}

Statistical analysis was performed by using the SAS-based statistical package JMP (www.jmp.com). For determination of the significance of improvement in the RDQ score, average pain during 24 hours, average pain with rest, and average pain with activity scores at days 1 and 3 were subtracted from the respective baseline scores. A paired $t$ test was performed with the null hypothesis being no difference in scores at days 1 and 3 compared with baseline. For comparison of outcome measures between patients enrolled in the LABEL study with patients enrolled in the control arm of the INVEST, we used 2-sample $t$ tests on the change from baseline scores. Means are presented with their associated SDs.

\section{Results}

\section{Patient Factors}

A total of 19 patients with 31 osteoporotic vertebral compression fractures were enrolled into the LABEL trial. Of these 19 patients, 8 were male and 11 were female. The mean age of the patients was $74.6 \pm 13.6$ years. Thirteen patients had 1 osteoporotic compression fracture, 2 patients had 2 osteoporotic compression fractures, 2 patients had 3 osteoporotic compression fractures, and 2 patients had 4 osteoporotic compression fractures. The levels treated were 1 T6, two T7, three T9, two T10, three T11, three T12, four L1, three L2, five L3, four L4, and 1 L5.

\section{Improvement in Function and Pain after Unblinded Lidocaine and Bupivacaine Injection (LABEL Outcomes)} Among the 19 patients enrolled into LABEL, the mean baseline severity for average pain during the past 24 hours, pain with activity, and pain at rest was $6.5 \pm 2.6,8.4 \pm 1.9$, and $3.6 \pm 2.5$, respectively (Table). At both days 1 and 3 postinjection, significant improvement compared with baseline was seen in pain with activity. For improvement in average pain during the past 24 hours, a trend toward significant improvement compared with baseline was noticed at days 1 and 3 . There was no significant improvement in pain at rest at days 1 and 3 compared with baseline. No significant improvement was noted in the RDQ scores because average improvement was $0.49 \pm 3.3$ at day 1 and $0.41 \pm 1.4$ at day $3(P=.82$ and $P=$ .32 at days 1 and 3 , respectively).

\section{Comparison of LABEL Patients with INVEST Control Patients}

Mean baseline severity for "average pain in the last 24 hours" for INVEST control patients at the lead site was $7.4 \pm 2.1$, not significantly different from that of LABEL patients $(P=.27)$. 


\begin{tabular}{|c|c|c|c|}
\hline \multicolumn{4}{|c|}{ Comparison of baseline factors and outcome measures between unblinded LABEL patients and blinded INVEST control-arm patients } \\
\hline & $\operatorname{LABEL}(n=19)^{\mathrm{a}}$ & INVEST Control Arm $(n=16)^{\mathrm{b}}$ & $P$ Value \\
\hline Age, mean (SD) & $74.6(13.6)$ & $74.3(9.4)$ & 0.94 \\
\hline Sex, (No.) (\%) female & $11(57.9)$ & $12(75.0)$ & 0.48 \\
\hline \multicolumn{4}{|l|}{ RDO, mean (SD) } \\
\hline Baseline & $18.1(4.0)$ & $17.6(4.6)$ & 0.73 \\
\hline Day 1 improvement ${ }^{\mathrm{c}}$ & $0.49(3.3)$ & - & - \\
\hline Day 3 improvement & $0.41(1.4)$ & $5.3(5.8)$ & 0.006 \\
\hline \multicolumn{4}{|c|}{ Average 24-hour pain, mean (SD) } \\
\hline Baseline & $6.5(2.6)$ & $7.4(2.1)$ & 0.27 \\
\hline Day 1 improvement & $1.4(2.9)$ & $3.8(3.2)$ & 0.03 \\
\hline Day 3 improvement & $0.72(1.4)$ & $2.9(3.4)$ & 0.04 \\
\hline
\end{tabular}

a In the LABEL study, 17/19 precrossover outcome measures were available at day 1, and 11/19 precrossover outcome measures, at day 3 .

${ }^{\mathrm{b}}$ For INVEST, outcome measures were available for all 16 patients at days 1 and 3.

${ }^{\mathrm{c}}$-indicates day $1 \mathrm{RDQ}$ scores for INVEST patients were not available.

INVEST control patients at the lead center demonstrated significantly greater improvement in average pain during the last 24 hours compared with the LABEL cohort at days 1 and 3 (Table). Mean baseline RDQ score for INVEST control patients at the lead site was $17.6 \pm 4.6$, which was not significantly different from that of LABEL patients $(P=.73)$. INVEST control patients demonstrated significantly greater improvement compared with baseline than those enrolled into LABEL (Table).

\section{Patient Crossover}

Of the 19 patients enrolled in this study, 16 (84\%) crossed over and underwent vertebroplasty and $3(16 \%)$ patients did not cross over by 3 months. The average number of days that patients who crossed over waited postinjection to receive their vertebroplasty was $4.3 \pm 3.8$ days (range, $1-14$ days; median, 3 days). Three patients crossed over the next day after the initial procedure, 3 patients crossed over on day 2, four patients crossed over on day 3 , four patients crossed over between days 4 and 7, and 2 patients crossed over between days 8 and 14 .

Patients who crossed over at day 3 or earlier $(n=10)$ had an average baseline RDQ score of $19.2 \pm 3.2$ and an average baseline 24-hour pain score of 6.9 \pm 2.3 . Patients who crossed over after day $3(n=9)$ had an average baseline RDQ score of $16.9 \pm 4.7$ and an average baseline 24-hour pain score of $6.0 \pm$ 3.0. There was no statistically significant difference in baseline RDQ scores $(P=.23)$ or baseline 24-hour pain between those patients who crossed over early and those who crossed over late $(P=.47)$.

For those patients who crossed over at day 3 or earlier, the average improvement in RDQ at day 1 was $0.1 \pm 2.2$ and the average improvement in 24 -hour pain was $2.2 \pm 3.8$. For those patients who crossed over after day 3 , the average improvement in RDQ at day 1 was $1.0 \pm 4.7$ and average improvement in 24 -hour pain at day 1 was $0.6 \pm 1.9$. At day 1 , there was no statistically significant difference in improvement in RDQ scores between the 2 groups $(P=.30)$ or improvement in 24-hour pain $(P=.61)$.

\section{Discussion}

In the current study, we demonstrated that patients undergoing an unblinded injection of local anesthesia for treatment of painful osteoporotic fractures achieve minimal improvement in pain. Depending on the specific pain question, maximum percentage improvement from baseline for LABEL patients ranged from $14 \%$ to $31 \%$, barely achieving the minimal clinically relevant difference of $30 \%$ improvement from baseline. ${ }^{7-9}$ Improvement in function as measured by the RDQ scale was both clinically and statistically insignificant as well. Notably, the observed improvement in both pain and function for LABEL patients was significantly less that that of INVEST patients, who underwent a similar infusion of local anesthesia in a blinded fashion. The observation of improvement in the setting of a blinded injection of local anesthesia that is significantly greater than that seen with identical injections without blinding suggests strongly that nonspecific factors, including the placebo effect, impacted observed improvement in the control arm of INVEST.

The mechanism of observed pain relief following vertebroplasty remains unknown, especially because the etiology of back pain following a vertebral body compression fracture is likely multifactorial. Potential mechanisms include cementmediated stabilization of microfractures as well as thermal and chemical damage to nerve endings. ${ }^{10-12}$ The lack of an observed dose-escalation effect, however, calls into question a direct effect of the infused cement. ${ }^{13}$ A recent histologic study of vertebral bodies in patients undergoing kyphoplasty found a paucity of nerve fibers located in the trabecular bone of the vertebral bodies, a finding that challenges the assertion that thermal or chemical damage to nerve endings is the reason for pain improvement. ${ }^{14}$ Commentaries on INVEST have postulated that the mechanism of pain improvement in the control group is due to factors such as breaking a cycle of pain through the prolonged effect of local anesthetic within the vertebral body. ${ }^{15,16}$ However, given the histologic evidence cited previously as well as the short (3.5 hours) half-life of bupivacaine, this seems unlikely. Thus, it appears as though the lack of improvement in the current LABEL trial would suggest strongly that nonspecific factors, including the placebo effect, were major determinants of improvement in the INVEST.

\section{Limitations}

This study had several important limitations. Most important, the sample size was small. Follow-up was limited because of the high rate of crossover to vertebroplasty; thus, we were able to show meaningful data only up to 3 days postinjection. We did not obtain systematic follow-up after the vertebroplasty procedures in these patients. Thus, we were unable to determine if vertebroplasty provided any of these patients with significant pain relief; however, the focus of this study was to 
determine the effect of the unblinded local anesthesia on pain associated with osteoporotic vertebral compression fractures. Also, we did not ask patients to provide a reason for their decision to cross over. The results of LABEL suggest that crossover was likely due to inadequate pain relief; however, it is possible that we may have missed other factors, such as expectation of increased benefit from vertebroplasty.

In comparing the 2 protocols of INVEST and LABEL, the important differences must be emphasized. As mentioned previously, with the injection of lidocaine and bupivacaine in INVEST control patients, patients were placed in an environment in which they were made to think they were receiving vertebroplasty. The injections were accompanied by a verbal clue commenting on needle placement and cement injection with manual palpation of the area of the fracture to simulate needle placement. This was not the case in LABEL because patients were informed that they would be undergoing only injection of local anesthesia. With regard to follow-up, in INVEST, the study coordinators collecting follow-up pain and RDQ scores were blinded to the treatment allocation of the patient, whereas in LABEL, this was not the case. Last, in LABEL we asked patients questions regarding pain at rest and pain with activity; however, this was not the case in INVEST.

\section{Conclusions}

Patients undergoing an unblinded injection of local anesthesia achieved significantly less improvement in pain and function compared with patients undergoing a similar injection in blinded fashion in a separate trial. These findings suggest that the observed improvement in the control group in the INVEST resulted from nonspecific factors, including the placebo effect, rather than the impact of local anesthesia.

\section{Acknowledgments}

We thank Harry J. Cloft, MD, PhD; Kent R. Thielen, MD; Jonathan M. Morris, MD; John I. Lane, MD; Patrick H. Luet- mer, MD; Felix E. Diehn, MD; and John T. Wald, MD for their effort in recruiting and treating patients.

\section{References}

1. Buchbinder R, Osborne RH, Ebeling PR, et al. A randomized trial of vertebroplasty for painful osteoporotic vertebral fractures. N Engl J Med 2009;361: 557-68

2. Kallmes DF, Comstock BA, Heagerty PJ, et al. A randomized trial of vertebroplasty for osteoporotic spinal fractures. N Engl J Med 2009;361:569-79

3. Patrick DL, Deyo RA, Atlas SJ, et al. Assessing health-related quality of life in patients with sciatica. Spine (Phila Pa 1976) 1995;20:1899-908, discussion 1909

4. Roland M, Fairbank J. The Roland-Morris Disability Questionnaire and the Oswestry Disability Questionnaire. Spine (Phila Pa 1976) 2000;25:3115-24

5. Trout AT, Kallmes DF, Gray LA, et al. Evaluation of vertebroplasty with a validated outcome measure: the Roland-Morris Disability Questionnaire. AJNR Am J Neuroradiol 2005;26:2652-57

6. Gray LA, Jarvik JG, Heagerty PJ, et al. INvestigational Vertebroplasty Efficacy and Safety Trial (INVEST): a randomized controlled trial of percutaneous vertebroplasty. BMC Musculoskelet Disord 2007;8:126

7. Dworkin RH, Turk DC, Wyrwich KW, et al. Interpreting the clinical importance of treatment outcomes in chronic pain clinical trials: IMMPACT recommendations. J Pain 2008;9:105-21

8. Farrar JT, Young JP Jr, LaMoreaux L, et al. Clinical importance of changes in chronic pain intensity measured on an 11-point numerical pain rating scale. Pain 2001;94:149-58

9. Ostelo RW, Deyo RA, Stratford P, et al. Interpreting change scores for pain and functional status in low back pain: towards international consensus regarding minimal important change. Spine (Phila Pa 1976) 2008;33:90-94

10. Amar AP, Larsen DW, Esnaashari N, et al. Percutaneous transpedicular polymethylmethacrylate vertebroplasty for the treatment of spinal compression fractures. Neurosurgery 2001;49:1105-14, discussion 1114-15

11. Barr JD, Barr MS, Lemley TJ, et al Percutaneous vertebroplasty for pain relief and spinal stabilization. Spine (Phila Pa 1976) 2000;25:923-28

12. Hodler J, Peck D, Gilula LA. Midterm outcome after vertebroplasty: predictive value of technical and patient-related factors. Radiology 2003;227:662-68

13. Kaufmann TJ, Trout AT, Kallmes DF. The effects of cement volume on clinical outcomes of percutaneous vertebroplasty. AJNR Am J Neuroradiol 2006;27: 1933-37

14. Buonocore M, Aloisi AM, Barbieri M, et al. Vertebral body innervation: implications for pain. J Cell Physiol 2010;222:488-91

15. Bolster MB. Consternation and questions about two vertebroplasty trials. Cleve Clin J Med 2010;77:12-16

16. Orr RD. Vertebroplasty, cognitive dissonance, and evidence-based medicine: what do we do when the 'evidence' says we are wrong? Cleve Clin J Med 2010; 77:8-11 